\title{
The Natural Compound Withaferin A Covalently Binds to Cys239 of $\beta$-Tubulin to Promote Tubulin Degradation
}

\author{
Jianhong Yang, Wei Yan, Yong Li, Lu Niu, Haoyu Ye, and Lijuan Chen \\ State Key Laboratory of Biotherapy and Cancer Center, West China Hospital of Sichuan University and Collaborative Innovation \\ Center of Biotherapy and Cancer, Chengdu, People's Republic of China
}

Received July 10, 2019; accepted September 28, 2019

\section{ABSTRACT}

Withaferin $A(W I T)$ is a natural product possessing a wide range of pharmacologic activities. Previous studies have reported covalent binding of WIT to tubulin and down-of tubulin protein levels although the underlying mechanisms remain to be established. In the current investigation, we showed that WIT induces down-regulation of tubulin in a post-transcriptional manner, suggestive of direct and potent activity in tubulin degradation. The $N, N^{\prime}$-ethylene bis(iodoacetamide) assay and competitive binding experiments with four colchicine sitetargeted tubulin inhibitors further revealed that WIT interacts with the colchicine site of tubulin to promote degradation. WIT irreversibly inhibited tubulin polymerization, and mass spectrometry results disclosed binding to cysteine at position 239 (Cys239) and Cys303 sites of $\beta$-tubulin. Interestingly, WIT promoted degradation of the $\beta$-tubulin isoforms containing Cys239 $[\beta 2, \beta 4$, and $\beta 5(\beta)]$ but had no effect on those containing Ser239 ( $\beta 3$ and $\beta 6$ ). Moreover, a C239S but not C303S mutation in $\beta$-tubulin completely abolished the degradation effect of WIT, suggesting that the Cys239-WIT covalent bond accounts for this activity. Our collective results clearly demonstrate that covalent interactions between WIT and Cys239 of $\beta$-tubulin promote tubulin degradation, supporting its potential utility as a therapeutic compound.

\section{SIGNIFICANCE STATEMENT}

Withaferin $A$, a natural product possessing a wide range of pharmacologic activities, covalently binds to Cys239 of $\beta$-tubulin near the colchicine site, and the WIT-Cys239 covalent bond accounts for WIT-induced tubulin degradation, fully clarifying the underlying mechanisms and supporting its potential utility a therapeutic compound.

\section{Introduction}

Withaferin A (WIT), a natural compound with a steroidal lactone structure isolated from Acnistus arborescens, Withania somnifera, and other species of the Solanaceae family (Kupchan et al., 1969; Mohan et al., 2004), has a long history of use as traditional medicine in India. Multiple pharmacologic activities of WIT have been identified to date, including cardioprotection, antidiabetic, anti-inflammatory, immune regulation, antiangiogenesis, and antitumor effects (Mohan et al., 2004; Maitra et al., 2009; Munagala et al., 2011; Gambhir et al., 2015; Ravindran et al., 2015; Lee et al., 2016; Tekula et al., 2018). The presence of active groups, such as a ketone-containing unsaturated A ring, epoxidecontaining $\mathrm{B}$ ring, and unsaturated lactone ring in WIT, imparts strong biologic activity (Gu et al., 2014; Siddique

This work was supported by the National Natural Science Foundation of China (81803021 and 81872900), China Postdoctoral Science Foundation (2019T120855, 2019M650248), Post-doctoral Research Project, West China Hospital, Sichuan University (2018HXBH027), Sichuan Science and Technology Program (2019YJ0088), and the 1.3.5 project for disciplines of excellence, the West China Hospital, Sichuan University.

https://doi.org/10.1124/mol.119.117812. et al., 2014) through interactions with specific amino acid residues in target proteins to form covalent bonds. For example, an unsaturated $\alpha, \beta$-double bond in an A ring has been shown to covalently bind cysteine through a Michael addition reaction. Additionally, the epoxide ring can react with a histidine, serine, or threonine residue to form a covalent bond (Drahl et al., 2005). The double bond in ring A and epoxide in ring $\mathrm{B}$ are mainly responsible for cytotoxicity (Vanden Berghe et al., 2012).

Plurality of target proteins corresponding to the pharmacologic activities of WIT has been reported. WIT reacts covalently with a cysteine residue in the majority of these targets, including cysteine 328 (Cys328) of vimentin (Bargagna-Mohan et al., 2007), Cys 179 of $\mathrm{I}_{\kappa} \mathrm{B}$ kinase $\beta$ (IKK $\beta$ ) (Heyninck et al., 2014), Cys133 of annexin II (Falsey et al., 2006), and a certain Cys at the N-terminus of heat shock protein 90 (HSP90) (Yu et al., 2010), highlighting its potential utility as a multitarget bioactive compound.

Microtubules, tubular structures formed by the assembly of $\alpha \beta$-tubulin in a head-to-tail structure (Stanton et al., 2011), are a popular target for the development of antitumor drugs. For example, the commonly used chemotherapeutic drugs

ABBREVIATIONS: Cl, confidence interval; Cys, cysteine; DWIT, dihydrowithaferin A; EBI, $N, N^{\prime}$-ethylenebis(iodoacetamide); GAPDH, glyceraldehyde-3-phosphate dehydrogenase; GFP, green fluorescent protein; MG132, benzyl $N$-[(2S)-4-methyl-1-[[(2S)-4-methyl-1-[[(2S)-4-methyl-1oxopentan-2-yl]amino]-1-oxopentan-2-yl]amino]-1-oxopentan-2-yl]carbamate; MS/MS, tandem mass spectrometry; PBST, PBS containing $0.1 \%$ Tween-20; PCR, polymerase chain reaction; PROTAC, proteolysis targeting chimera; PVDF, polyvinylidene difluoride; WIT, Withaferin A; WT, wild type. 
paclitaxel, vinblastine, and eribulin mesylate (Halavan) exert their activities by targeting microtubules (Dumontet and Jordan, 2010). Microtubules play important roles in diverse physiologic activities of cells, including motility, intracellular protein transport, and cell division (Stanton et al., 2011). The antitumor drugs targeting microtubules mainly inhibit tumor cell growth by suppressing cell division (Stanton et al., 2011). Six binding sites on the tubulin dimer have been identified for paclitaxel (Ojeda-Lopez et al., 2014), vinblastine (Gigant et al., 2005), colchicine (Ravelli et al., 2004), maytansine (Prota et al., 2014a), laulimalide (Prota et al., 2014b), and pironetin (Yang et al., 2016), respectively.

Small molecules that bind different sites induce microtubule inhibition through distinct mechanisms. Compounds binding to paclitaxel or laulimalide sites promote tubulin polymerization while interactions with the other four sites lead to inhibition of polymerization ( $\mathrm{Na}$ and Timasheff, 1982; Yang et al., 2016). Vinblastine-binding compounds display a unique feature in that they induce the formation of tubulin paracrystals (packing of unpolymerized tubulin in the cytoplasm) (Gigant et al., 2005). These diverse mechanisms of tubulin inhibition provide several options for microtubule drug development and facilitate comprehensive investigation of microtubule polymerization and depolymerization processes.

Earlier, Antony et al. (2014) reported that WIT inhibits tubulin through covalent binding to a novel site at Cys303 of $\beta$-tubulin, (Antony et al., 2014) and exerts a degradation effect distinct from other conventional tubulin inhibitors. However, it remains to be established whether WIT-Cys303 binding is specific and degradation of tubulin is directly related to interactions with WIT, as Cys303 is located on the surface of $\beta$-tubulin. More importantly, we have yet to ascertain whether covalent bond formation between WIT and Cys303 is the direct cause of tubulin degradation. To identify potential novel tubulin-binding sites, we conducted an in-depth study of the interactions between WIT and tubulin. Our data showed that WIT forms covalent bonds with Cys303 and Cys239 of $\beta$-tubulin. Moreover, the bond between WIT and Cys239, but not Cys303, was responsible for tubulin degradation, providing another example of a small molecule that acts as a covalent modifier of Cys239 in $\beta$-tubulin to promote tubulin degradation (Yang et al., 2019).

\section{Materials and Methods}

Reagents. Colchicine, paclitaxel, vinblastine, nocodazole, plinabulin, combretastatin A4, N,N'-ethylenebis(iodoacetamide) (EBI), and benzyl $\quad N$-[(2S)-4-methyl-1-[[(2S)-4-methyl-1-[[(2S)-4-methyl-1-oxopentan-2-yl]amino]-1-oxopentan-2-yl] amino]-1-oxopentan-2-yl]carbamate (MG132) were purchased from Selleck Chemicals (Houston, TX), WIT from Sigma-Aldrich (St. Louis, MO), and dihydrowithaferin A (DWIT) from BioCrick Biotech (Chengdu, Sichuan, People's Republic of China). Other conventional agents were obtained from SigmaAldrich or Kelun Pharmaceuticals (Chengdu, Sichuan, People's Republic of China). Purified tubulin was acquired from Cytoskeleton Inc. (Denver, CO), and all antibodies were purchased from Abcam (Cambridge, MA): glyceraldehyde-3-phosphate dehydrogenase (GAPDH): ab181602; $\beta$-actin: ab179467; $\alpha$-tubulin: ab7291; $\beta$-tubulin: ab6046; $\beta 2$-tubulin: ab151318; $\beta 3$-tubulin: ab52623; $\beta 4$-tubulin: ab179509; $\beta 6$ tubulin: ab110592; p-H3 (phosphorylation at ser10): ab5176.

Cell Lines and Cultures. HeLa and Hct116 cells were sourced from the American Type Culture Collection (Manassas, VA). Both cell lines were cultured in Dulbecco's modified Eagle's medium supplemented with fetal bovine serum (10\%) and double antibiotics: penicillin $(100 \mathrm{U} / \mathrm{ml})$ and streptomycin $(100 \mu \mathrm{g} / \mathrm{ml})$. Cells were cultured at $37^{\circ} \mathrm{C}$ with $5 \% \mathrm{CO}_{2}$ in a humidified chamber.

Trypan Blue Exclusion. Cells were plated into six-well plates and cultured for 24 hours. After treatment with the specified compounds, the cells were incubated for a further 48 hours. Both alive and dead cells were collected, washed with PBS, and then stained with trypan blue dye $(0.4 \mathrm{mg} / \mathrm{ml}$ in PBS) for 6 minutes. Then cells were counted with a hemocytometer to calculate the death ratio.

Western Blot. Cells in six-well plates were harvested after treatment with the specified compounds and washed with PBS for 2 minutes. The collected cells were lysed with radioimmunoprecipitation assay buffer containing $5 \times$ loading buffer for 10 minutes with manual vibration every 2 minutes. Lysed samples were subjected to denaturation in boiling water for 10 minutes and preserved at $-20^{\circ} \mathrm{C}$ until experimental use. Equal amounts of samples $(20 \mu \mathrm{g})$ were subjected to SDS-PAGE for separation and proteins on gels transferred to polyvinylidene difluoride (PVDF) membranes.

The PVDF membranes were incubated with primary antibody for 12 hours at $4^{\circ} \mathrm{C}$ and washed with PBST (PBS containing $0.1 \%$ Tween$20)$ buffer for 30 minutes $(3 \times 10$ minutes $)$ before incubation with secondary antibody for 45 minutes at room temperature. The PVDF membranes were rewashed with PBST buffer for 30 minutes, subjected to immunoreactivity analysis and visualized under a chemiluminescence imaging system (Tanon, Shanghai, People's Republic of China).

EBI Assay. Purified tubulin $(1 \mu \mathrm{M})$ was incubated with the specified compounds for 2 hours and with $100 \mu \mathrm{M}$ EBI for a further 2 hours. Samples were mixed with $5 \times$ loading buffer and denatured in boiling water for 10 minutes. Equal amounts of samples were analyzed via Western blot using $\beta$-tubulin antibody.

Mass Spectrometry. Purified $\alpha \beta$-tubulin dimer $(10 \mu \mathrm{M})$ and excess WIT $(30 \mu \mathrm{M})$ were incubated for 3 hours at room temperature. Unreacted WIT was removed via ultrafiltration. The samples were mixed with loading buffer and loaded on to SDS gels for electrophoresis, followed by staining with Coomassie brilliant blue. The tubulin band was excised, digested with trypsin, and subjected to mass spectrometry analysis according to our previously published protocol (Yang et al., 2016).

Immunofluorescence Staining. Cells on coverslips in 24-well plates were treated with high concentrations of compounds $(10 \mu \mathrm{M}$ paclitaxel, $10 \mu \mathrm{M}$ colchicine, $10 \mu \mathrm{M}$ vinblastine, and $100 \mu \mathrm{M}$ WIT) for 1 hour. After removal of the supernatant, the cells were washed with PBS for 2 minutes before fixing with 50\% methanol/50\% acetone for 2 minutes. Next, the cells were incubated with tubulin antibody at $4^{\circ} \mathrm{C}$ overnight and washed with PBST for 20 minutes $(4 \times 5$ minutes $)$. After further incubation with secondary antibody and 4',6-diamidino-2phenylindole (DAPI) for 45 minutes at room temperature, the cells were rewashed with PBST for 20 minutes $(4 \times 5$ minutes). The prepared samples were observed and imaged under an Olympus fluorescence microscope (Tokyo, Japan).

In Vitro Tubulin Polymerization Assay. The in vitro tubulin polymerization assay was performed according to the established protocol of Cytoskeleton, Inc. (https://www.cytoskeleton.com/pdfstorage/datasheets/bk006p.pdf). Briefly, purified tubulin $(3 \mathrm{mg} / \mathrm{ml})$ was incubated with the specified compounds at $4^{\circ} \mathrm{C}$ for 1 minute before transfer to a precold microplate reader (BioTek Instruments, Winooski, VT). Optical density values at $340 \mathrm{~nm}$ were measured once per minute.

Quantitative Polymerase Chain Reaction. Cells in six-well plates were treated with WIT for 16 hours, then collected and washed with PBS. The total mRNA was obtained with TRIzol reagent (Invitrogen, Carlsbad, CA) according to the manufacturer's protocol. A high-capacity cDNA reverse transcription kit (Applied Biosystems, Foster City, CA) was employed for reverse transcription of mRNA to DNA. Quantitative polymerase chain reaction (PCR) detection of $\alpha$-and $\beta$-tubulin levels was conducted following our previously established protocols (Yang et al., 2019). 
Vector Construction. The full-length wild-type (WT) TUBB gene sequence containing the FLAG-tag at the C-terminus was synthesized by Genewiz (Suzhou, People's Republic of China). The gene was cloned into MSCV-IRES-GFP vector for coexpression of the target protein and green fluorescent protein (GFP). Genes containing the C239S and C303S mutations were constructed using the Q5 site-directed mutagenesis kit (E0554S; New England Biolabs, Ipswich, MA).

Transfection Experiments. HeLa cells were transiently transfected with the MSCV-IRES-GFP vector containing WT FLAG- $\beta$-tubulin, C239S FLAG- $\beta$-tubulin, or C303S FLAG$\beta$-tubulin for 24 hours and treated with WIT for 16 hours. Total protein was obtained and analyzed via Western blot using FLAG and GFP antibodies. Expression of GAPDH was detected as a loading control.

Statistical Analysis. Statistics results of multiple experiments were shown as mean $\pm 95 \%$ confidence intervals (CI). Statistical differences were determined using an unpaired, nonparametric Student's $t$ test (Welch's). $* P<0.05$ was considered statistically significant.

\section{Results}

WIT Promotes Tubulin Degradation in a Post-transcriptional Manner. WIT (Fig. 1A) has been shown to suppress the protein levels of tubulin (Antony et al., 2014), but the exact mechanism remains unclear at present. Western blot findings revealed that WIT down-regulates $\alpha$-and $\beta$-tubulin proteins in a concentration-dependent manner in HeLa and HCT116 cells (Fig. 1B). However, simultaneous quantitative PCR experiments disclosed no inhibitory effects of WIT on mRNA levels of $\alpha$ - and $\beta$-tubulin (Fig. 1C), indicating that WIT-induced down-regulation is a post-transcriptional process. Conversely, WIT slightly promoted tubulin mRNA levels (Fig. 1C), which may be a result of negative regulation after tubulin protein suppression.

Immunofluorescence results indicated that WIT could also inhibit tubulin polymerization in HeLa cells at concentrations that promote tubulin degradation (Fig. 1D), indicating the tubulin degradation and depolymerization activities induced

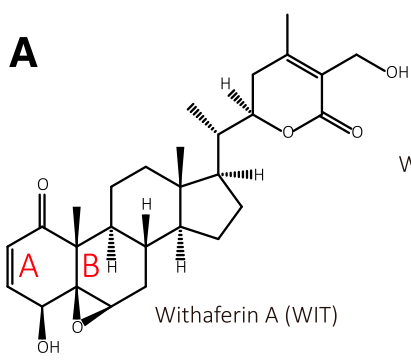

B
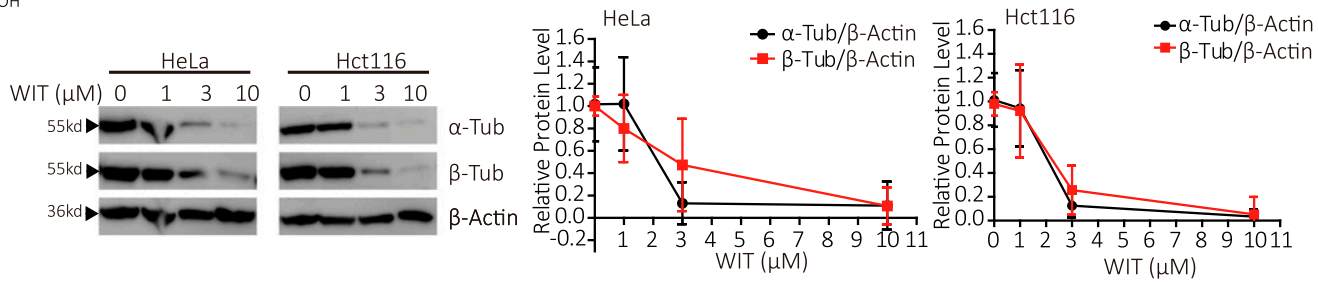

C

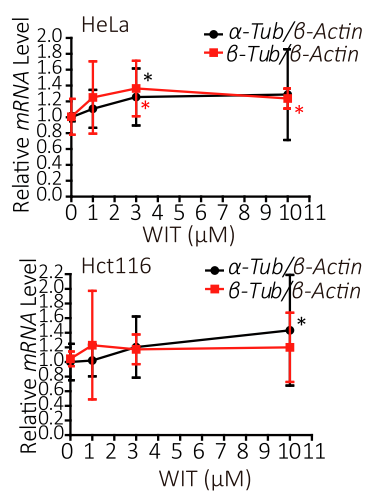

D
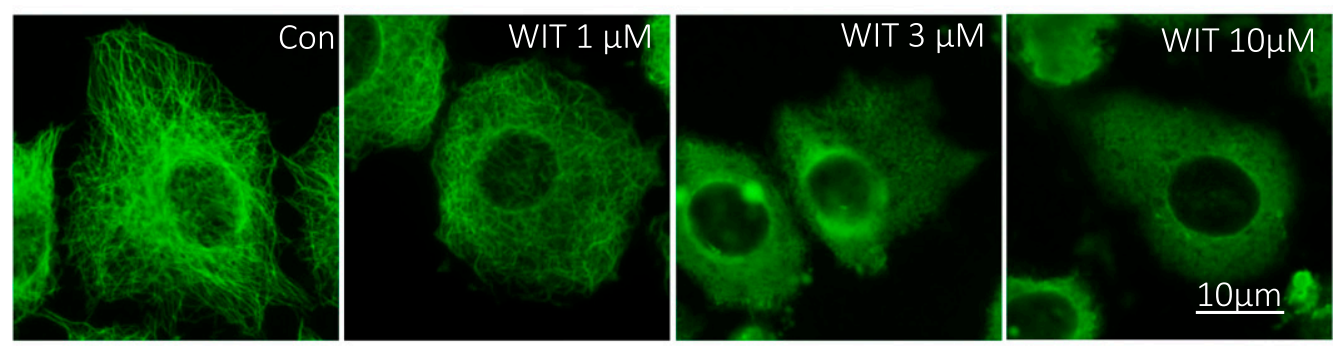

E

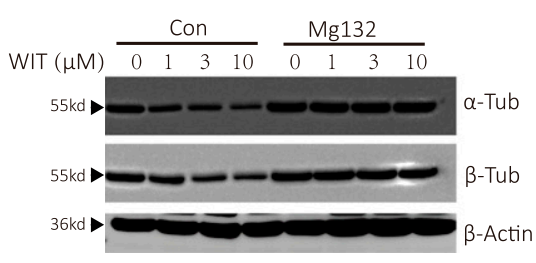

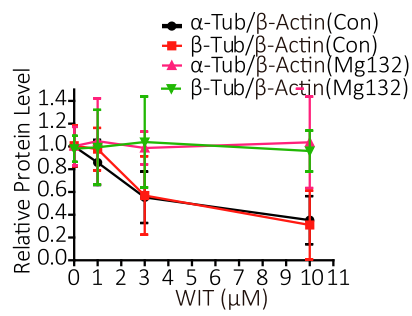

$\mathbf{F}$

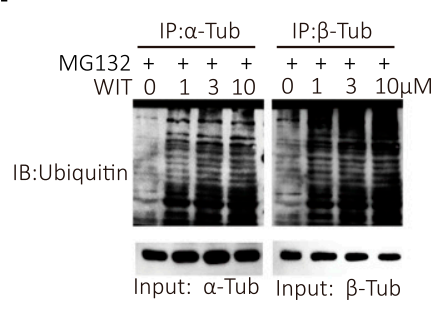

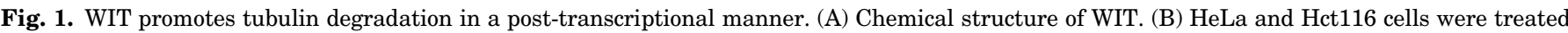

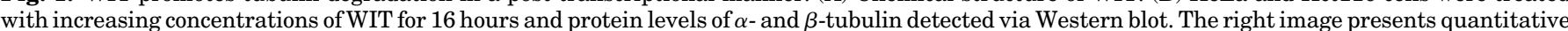

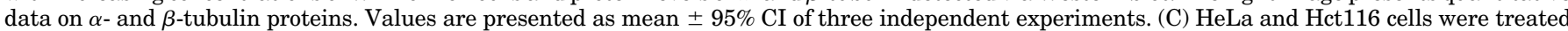

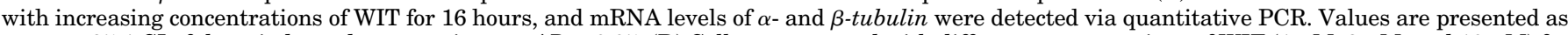

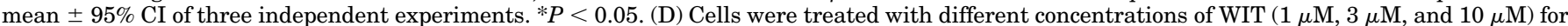

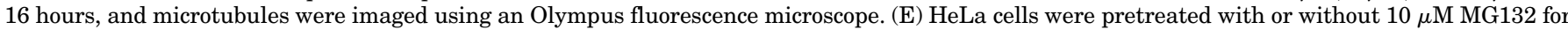

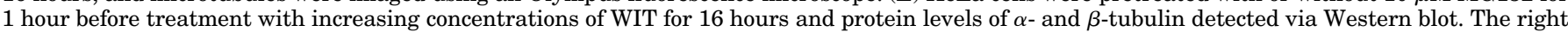

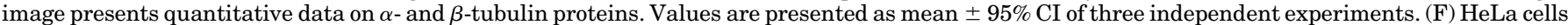

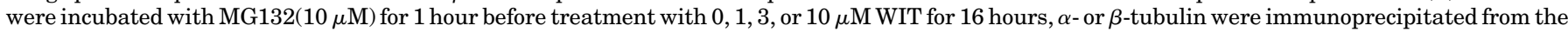

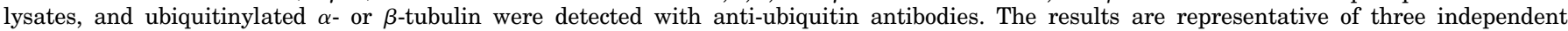
experiments. $\alpha$-Tub, $\alpha$-tubulin; $\beta$-Tub, $\beta$-tubulin; Con, Control. 
by WIT is initiated at almost identical concentration range. Upon pretreatment with the proteasome inhibitor MG132, WIT no longer induced down-regulation of tubulin (Fig. 1E). In addition, immunoprecipitation results revealed that WIT could increase the ubiquitination level of both $\alpha$ - and $\beta$-tubulin (Fig. 1F). All these results confirmed WIT induces tubulin protein degradation in a ubiquitin-proteasome (post-transcriptional) dependent manner.

WIT Binds to the Colchicine Site of $\boldsymbol{\beta}$-Tubulin. All the tubulin inhibitors identified to date bind tubulin to promote or inhibit microtubule polymerization. For instance, paclitaxel acts as a tubulin polymerization agent while colchicine and vinblastine are tubulin depolymerization agents (Dumontet and Jordan, 2010). Here, we investigated the effect of WIT on polymerization of tubulin in vitro using paclitaxel and colchicine as positive controls.

As shown in Fig. 2A, paclitaxel promoted polymerization of tubulin while colchicine exerted the opposite effect to a significant extent. WIT exerted an inhibitory effect on polymerization similar to that of colchicine, which was concentrationdependent, confirming direct interactions with tubulin.

Colchicine and vinblastine sites are the two most commonly studied tubulin depolymerization sites in $\beta$-tubulin, and interacting compounds at the two sites have distinct effects on microtubule morphology. At high concentrations, compounds at the vinblastine site induce depolymerization of tubulin to further form paracrystals (packing of unpolymerized tubulin in the cytoplasm) that can be detected using immunofluorescence. However, compounds binding to the colchicine site have no such effect.

Immunofluorescence results depicted in Fig. 2B showed that high concentrations of paclitaxel markedly promoted tubulin polymerization, colchicine inhibited tubulin polymerization, and vinblastine inhibited tubulin polymerization and significantly induced formation of tubulin paracrystals. Notably, the morphologic changes of tubulin depolymerization caused by high concentrations of WIT were similar to those of colchicine, indicative of WIT binding at the colchicine site. Accordingly, we further investigated whether WIT interacts with the colchicine site with the aid of the EBI competition binding assay.

One molecule of EBI is capable of simultaneously forming covalent bonds with cysteine residues at positions 239 and 354 near the $\beta$-tubulin colchicine site, thereby forming an EBI- $\beta$-tubulin complex that migrates faster than $\beta$-tubulin on SDS-PAGE (Fortin et al., 2010). As shown in Fig. 2C, both colchicine and WIT inhibited formation of the EBI- $\beta$-tubulin complex, supporting the

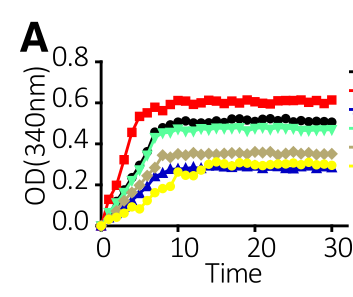

c
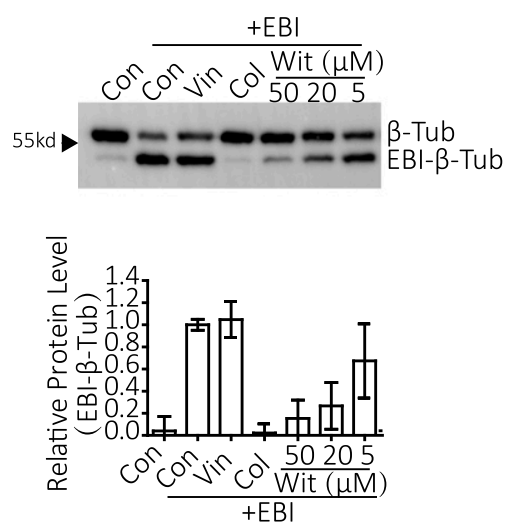
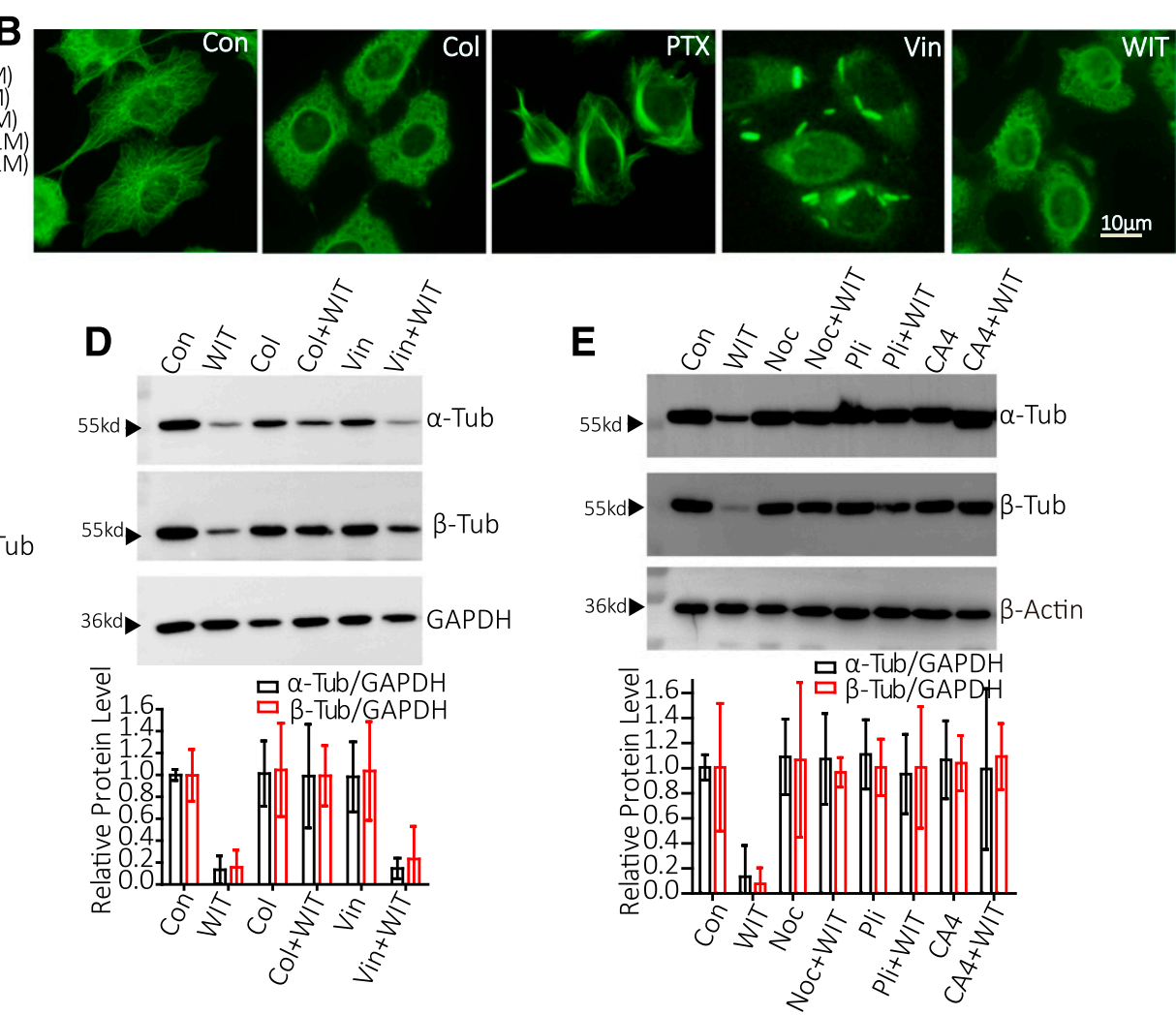

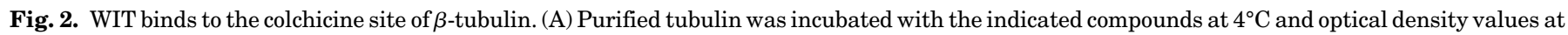

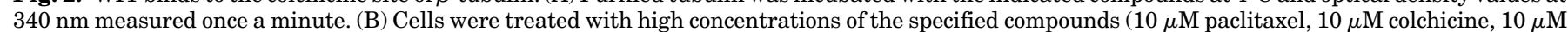

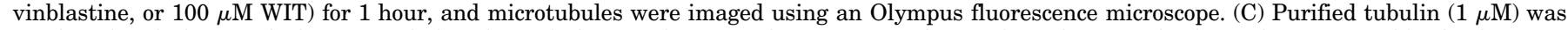

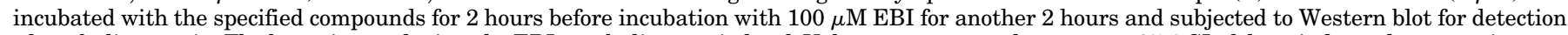

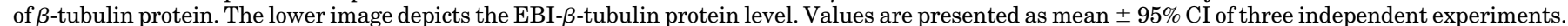

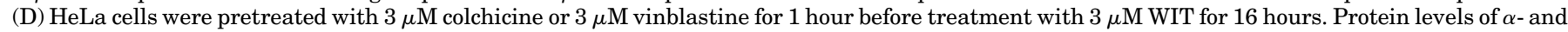

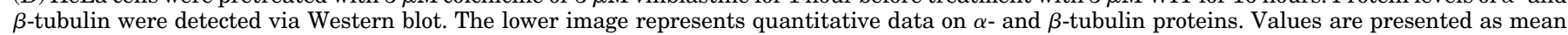

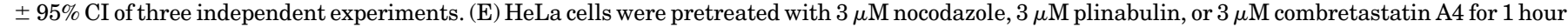

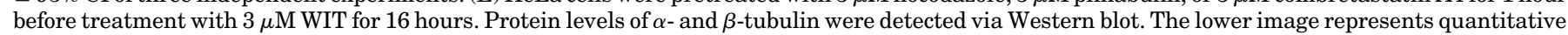

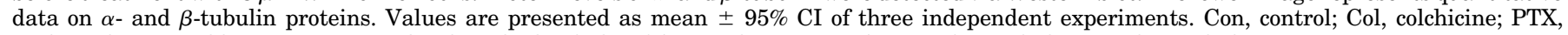
paclitaxel; Vin, vinblastine; Noc, nocodazole; Pil, plinabulin; CA4, combretastatin A4; $\alpha$-Tub, $\alpha$-tubulin; $\beta$-Tub, $\beta$-tubulin. 
theory that WIT interacts with the colchicine site of $\beta$-tubulin.

In competition experiments, pretreatment with colchicine, but not vinblastine, inhibited tubulin degradation by WIT (Fig. 2D), confirming that binding of WIT to the colchicine site accounts for tubulin degradation. To further validate this finding, we used a variety of colchicine site inhibitors, including nocodazole, plinabulin, and combretastatin A4, for another competition experiment. Our data showed significant inhibition of WIT-induced tubulin degradation by these inhibitors (Fig. 2E). The collective results indicate that WIT binds the colchicine site of tubulin to promote degradation.

WIT Exerts Irreversible Cellular Effects. Reversibility of binding to tubulin plays an important role in determining the toxicity and effectiveness of microtubule inhibitors (Thomas et al., 2014; Yang et al., 2018). Generally, reversible inhibitors exert lower toxicity and irreversible inhibitors have better efficacy. Simultaneous reversibility can also be used to predict the mechanisms by which inhibitors bind to tubulin. For example, an irreversible mode of action such as that of cyclostreptin usually indicates covalent binding to tubulin (Buey et al., 2007; Balaguer et al., 2019). Here, we examined the reversibility of WIT action in HeLa cells using the irreversible inhibitor colchicine and reversible inhibitor colcemid as positive controls (Yan et al., 2018; Yang et al., 2018).

After treatment with inhibitors for 8 hours, HeLa cells were washed and cultured for an additional 24 hours. Cell microtubule morphology was detected via immunofluorescence at the 0-, 8-, 16-, 24-, and 32-hour time points. As shown in Fig. 3A, at the 0 -hour time point, microtubules exhibited a normal network in the cytoplasm of HeLa cells. After 8 hours of treatment with colchicine, colcemid, or WIT, microtubule depolymerization was significantly enhanced and no polymerized microtubules were observed in the cytoplasm.

All inhibitors were totally removed at the 8-hour time point. At 16 hours, the colchicine and WIT-treated microtubes remained in a depolymerized state while colcemid-treated microtubules had begun to resume polymerization. Colcemidtreated microtubes continued to exhibit polymerization at the 24-hour time point while colchicine and WIT-treated tubulin remained in a depolymerized state. At the time point of 32 hours, the morphology of the microtubules in the colcemid treatment group had completely recovered to normal, but no significant differences were observed in colchicine-treated or WIT-treated microtubules between the time points of 8 and 32 hours. Simultaneous detection of the level of p-H3 on ser-10 (a G2/M phase arrest marker) (Ren et al., 2018) at the above time points revealed similar results (Fig. 3B).

These findings indicate that WIT behaves in a similar manner to colchicine as an irreversible microtubule inhibitor. Given its ability to form covalent bonds with target proteins, we further assessed whether WIT could bind covalently to the colchicine site of tubulin.

WIT Covalently Binds to Cys303 and Cys239 of $\boldsymbol{\beta}$-Tubulin. A biomass spectrometry experiment was performed to determine the specific residues forming covalent bonds with WIT on $\beta$-tubulin. Purified $\alpha \beta$-tubulin dimer $(10 \mu \mathrm{M})$ and excess WIT $(30 \mu \mathrm{M})$ were incubated for 3 hours at room temperature followed by removal of unbound WIT via ultrafiltration and SDS-PAGE. A 55-kD tubulin band was obtained, which was excised and subjected to mass spectrometry analysis.

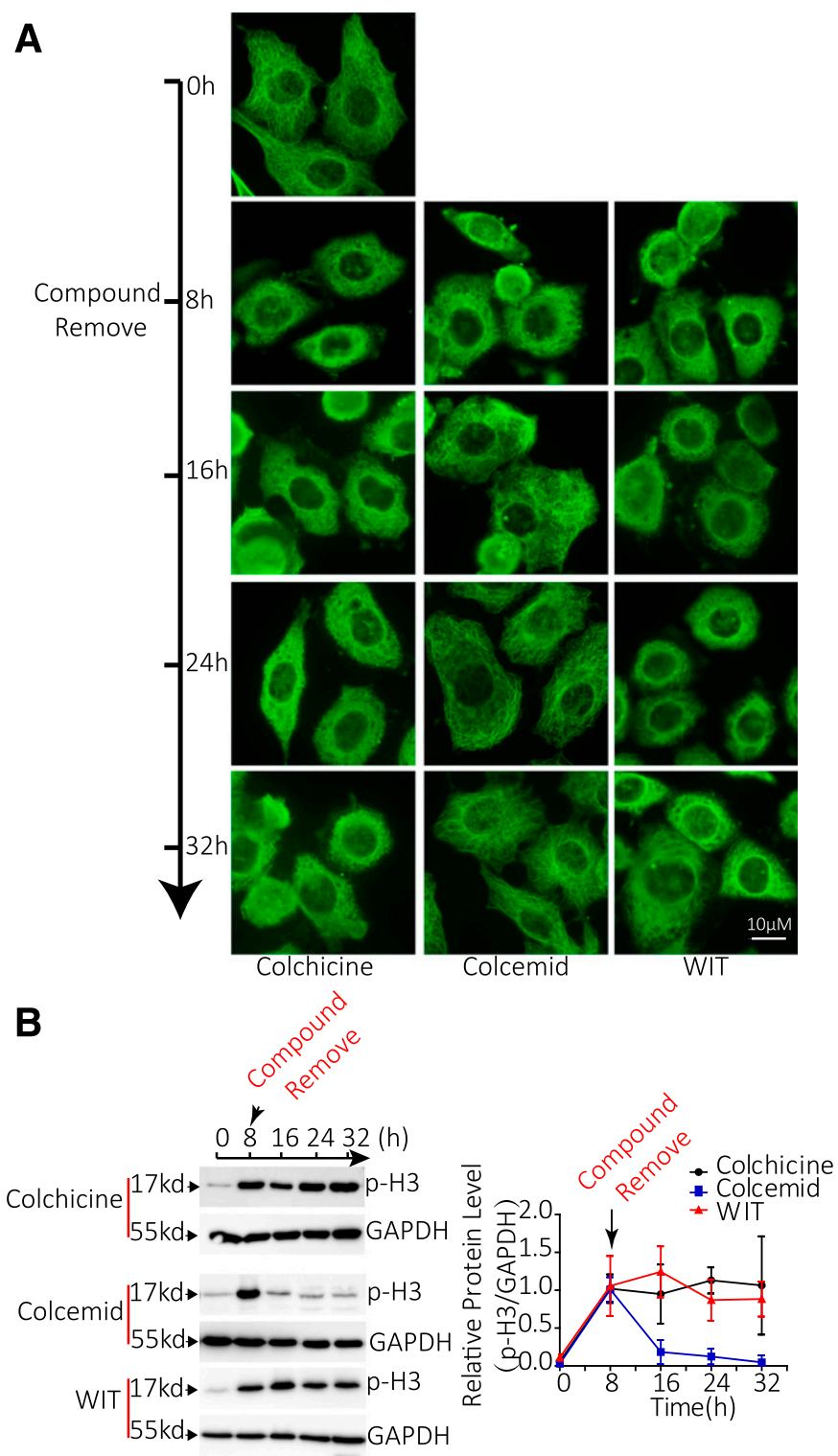

Fig. 3. WIT exerts irreversible cellular effects. (A) HeLa cells were treated with $10 \mu \mathrm{M}$ colchicine, $10 \mu \mathrm{M}$ colcemid, or $100 \mu \mathrm{M}$ WIT for 8 hours. After the compounds were completely washed off, the cells were cultured for an additional 24 hours, and their microtubule morphology was examined via immunofluorescence at the 0-, 8-, 16-, 24-, and 32-hour time points. (B) Western blot analysis of $\mathrm{p}-\mathrm{H} 3$ expression was further examined at $0,8,16$, 24 , and 32 hours. The right image depicts quantitative data on p-H3 expression. Values are presented as mean $\pm 95 \% \mathrm{CI}$ of three independent experiments.

The data showed that two peptides were covalently modified by WIT $\left({ }^{217}\right.$ LTTPTYGDLNHLVSATMSGVTTCLR ${ }^{241}$ and ${ }^{298} \mathrm{NMMAACDPR}^{306}$ ) in $\beta$-tubulin with a molecular weight increase of $470 \mathrm{kD}$ (molecular weight of WIT), respectively. The untreated peptides showed only a weight increase of 57 $\mathrm{kD}$ (carbamidomethyl). The tandem mass spectrometry (MS/ MS) fragment of the WIT-treated ${ }^{217}$ LTTPTYGDLNHLVSATMSGVTTCLR ${ }^{241}$ peptide showed that all y-ions containing Cys239 were modified by WIT, supporting covalent binding to Cys239 (Fig. 4A). The ${ }^{298}$ NMMAACDPR ${ }^{306}$ peptide fragmentation data similarly revealed that WIT forms a covalent bond with Cys303 (Fig. 4B). Our results collectively demonstrate that one molecule of $\beta$-tubulin can simultaneously 
form covalent bonds with two molecules of WIT at the Cys239 and Cys303 sites.

Covalent Binding between WIT and Cys239 Accounts for WIT-Induced Tubulin Degradation. Multiple subtypes of tubulin exist, including $\beta 2-, \beta 3-, \beta 4-, \beta 5(\beta)$ - and $\beta 6$ tubulin (Ernest, 2008). Among these isoforms, $\beta 3$ - and $\beta 6$ tubulin contain Ser while $\beta 2-, \beta 4-$, and $\beta 5$ - $(\beta)$-tubulin contain Cys at position 239. All the tubulin isoforms contain Cys303 (Fig. 5A). In this study, we investigated the effects of WIT on $\beta 2-, \beta 3-, \beta 4-$, and $\beta 6$-tubulin protein levels.

Interestingly, WIT significantly promoted degradation of $\beta 2$ - and $\beta 4$-tubulin containing cysteine at position 239 but had no effect on $\beta 3$ - and $\beta 6$-tubulin levels (Fig. $5 \mathrm{~B}$ ), suggesting that covalent binding of WIT to tubulin Cys239 is the main factor underlying its degradation activity. For validating of this finding, FLAG-tagged (C-termini) WT $\beta$-tubulin or mutant $\beta$-tubulin (C239S and C303S) genes were cloned into MSCVIRES-GFP expression vectors, which coexpressed tubulin and GFP in HeLa cells after transient transfection. WIT promoted degradation of WT $\beta$-tubulin and C303S $\beta$-tubulin but had no effect on C239S $\beta$-tubulin (Fig. 5C), suggesting that only Cys239 is required for WIT activity on tubulin.

The unsaturated double bond in the A ring of WIT is liable to undergo a Michael addition reaction with the sulfhydryl group of cysteine (Falsey et al., 2006; Maitra et al., 2009; Yu et al., 2010; Antony et al., 2014). Accordingly, we further focused on the structure-activity relationship of WIT. DWIT is a structural analog of WIT (Khan et al., 2016), the only difference being the lack of an unsaturated double bond in ring $\mathrm{A}$ of DWIT (Fig. 5D). DWIT did not promote tubulin degradation (Fig. 5E) or inhibit cell viability in HeLa cells (Fig. 5F). Based on these results, we have concluded that covalent bond formation between WIT and Cys239 is responsible for tubulin degradation.

\section{Discussion}

Since 2007, the U.S. Food and Drug Administration has approved three antitumor drugs targeting microtubules, specifically, ixabepilone, cabazitaxel, and eribulin mesylate.
A

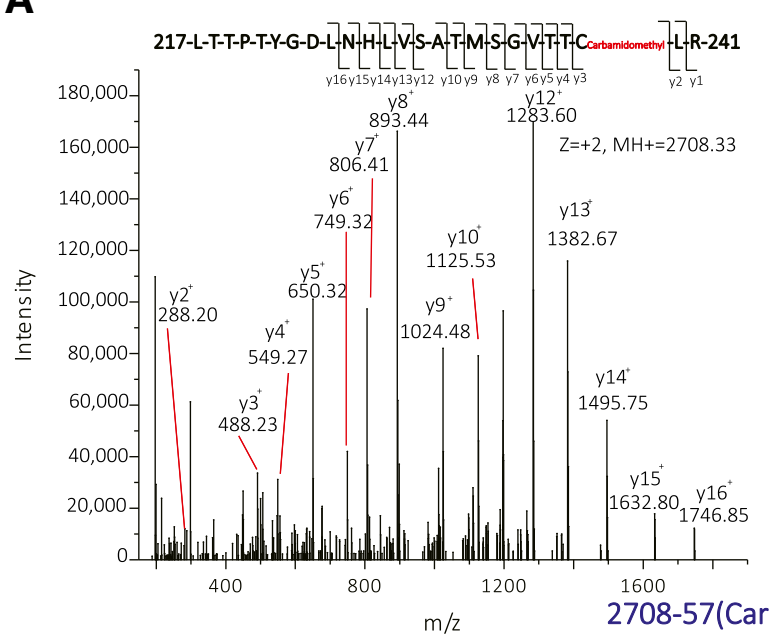

B

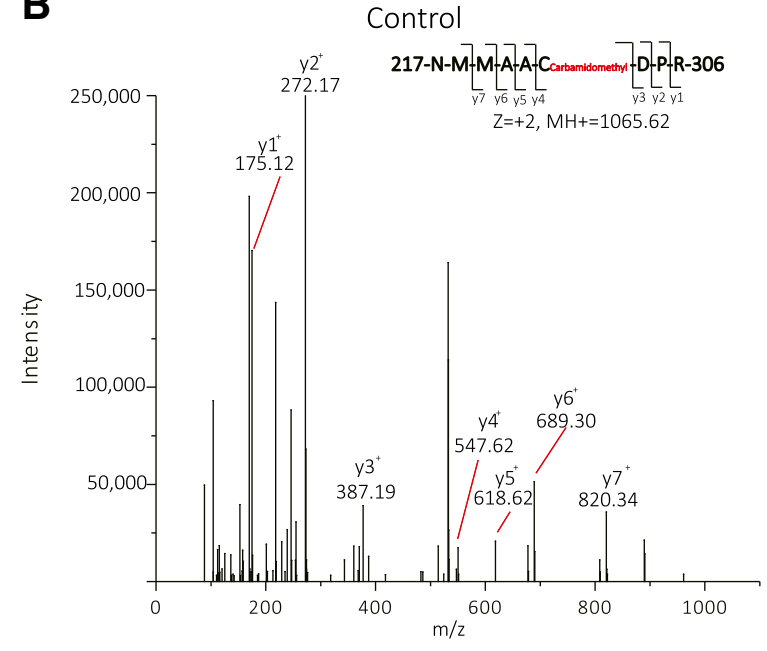

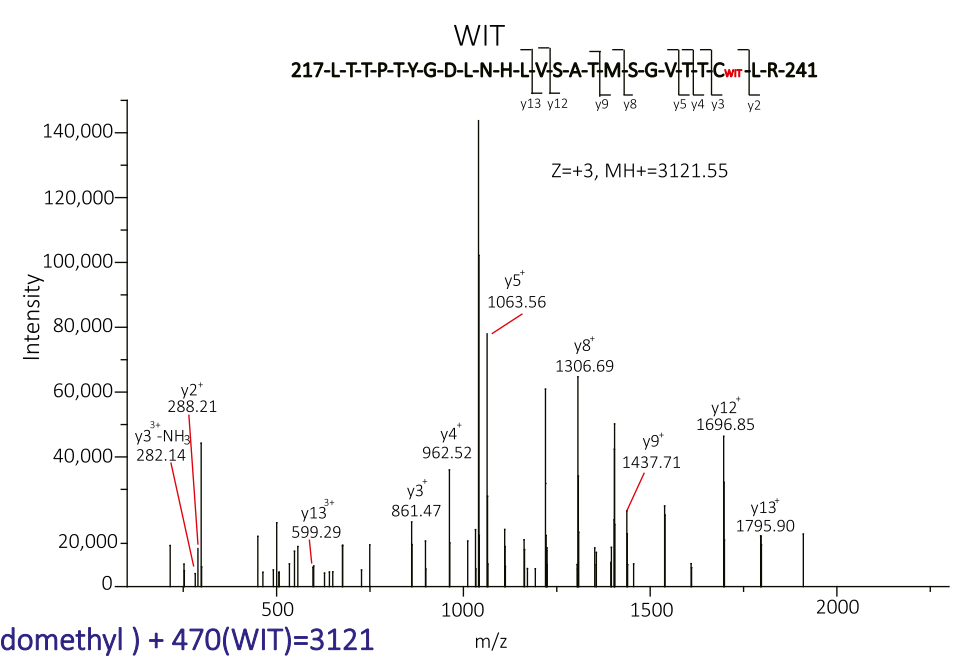

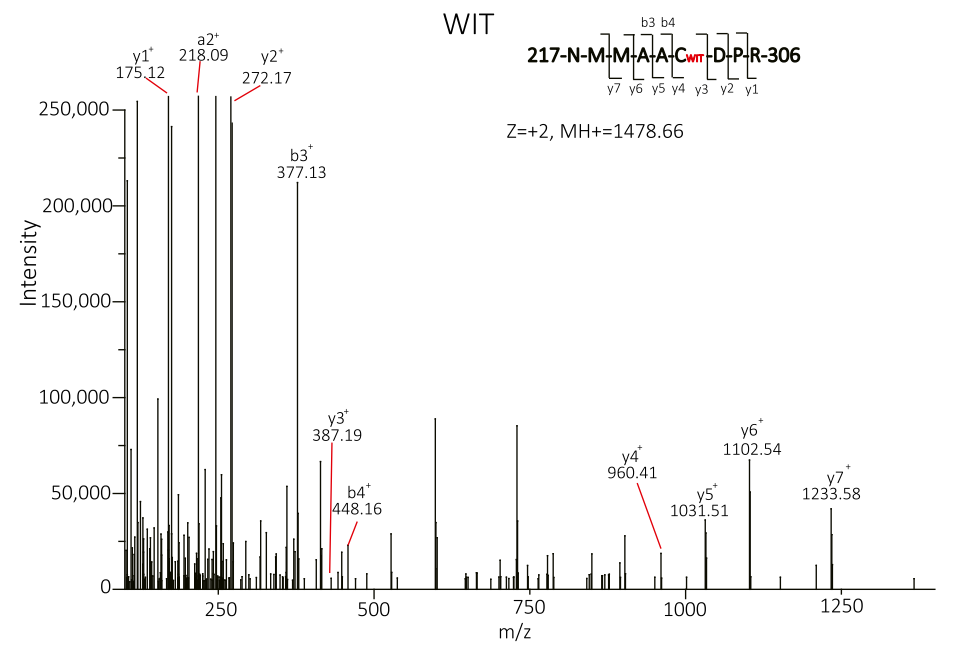

1065-57(Carbamidomethyl $)+470($ WIT $)=1478$

Fig. 4. WIT forms covalent bonds with Cys303 and Cys239 of $\beta$-tubulin. (A) Left: MS/MS fragmentation patterns of the untreated peptide ${ }_{217}$ LTTPTYGDLNHLVSATMSGVTTCLR ${ }^{241}$. Right: MS/MS fragmentation patterns of the covalently bound peptide 217 LTTPTYGDLNHLVSATMSGVTTCLR ${ }^{241}$ indicate that WIT binds to Cys239. (B) Left: MS/MS fragmentation pattern of the untreated peptide ${ }^{298}$ NMMAACDPR $^{306}$. Right: MS/MS fragmentation pattern of the covalently bound peptide ${ }^{298}$ NMMAACDPR $^{306}$ indicate that WIT binds to Cys303. 
A $\quad$ 2: 231 ATMSGVTTCL 240 B

B3: 231 ATMSGVTTSL 240

ß4: 231 ATMSGVTTCL 240

B5: 231 ATMSGVTTCL 240

$\beta 6: 231$ ATMSGVTTSL 240

B2: 301 AACDPRHGRY 310

B3: 301 AACDPRHGRY 310

B4: 301 AACDPRHGRY 310

B5: 301 AACDPRHGRY 310

B6: 301 AACDPRHGRY 310
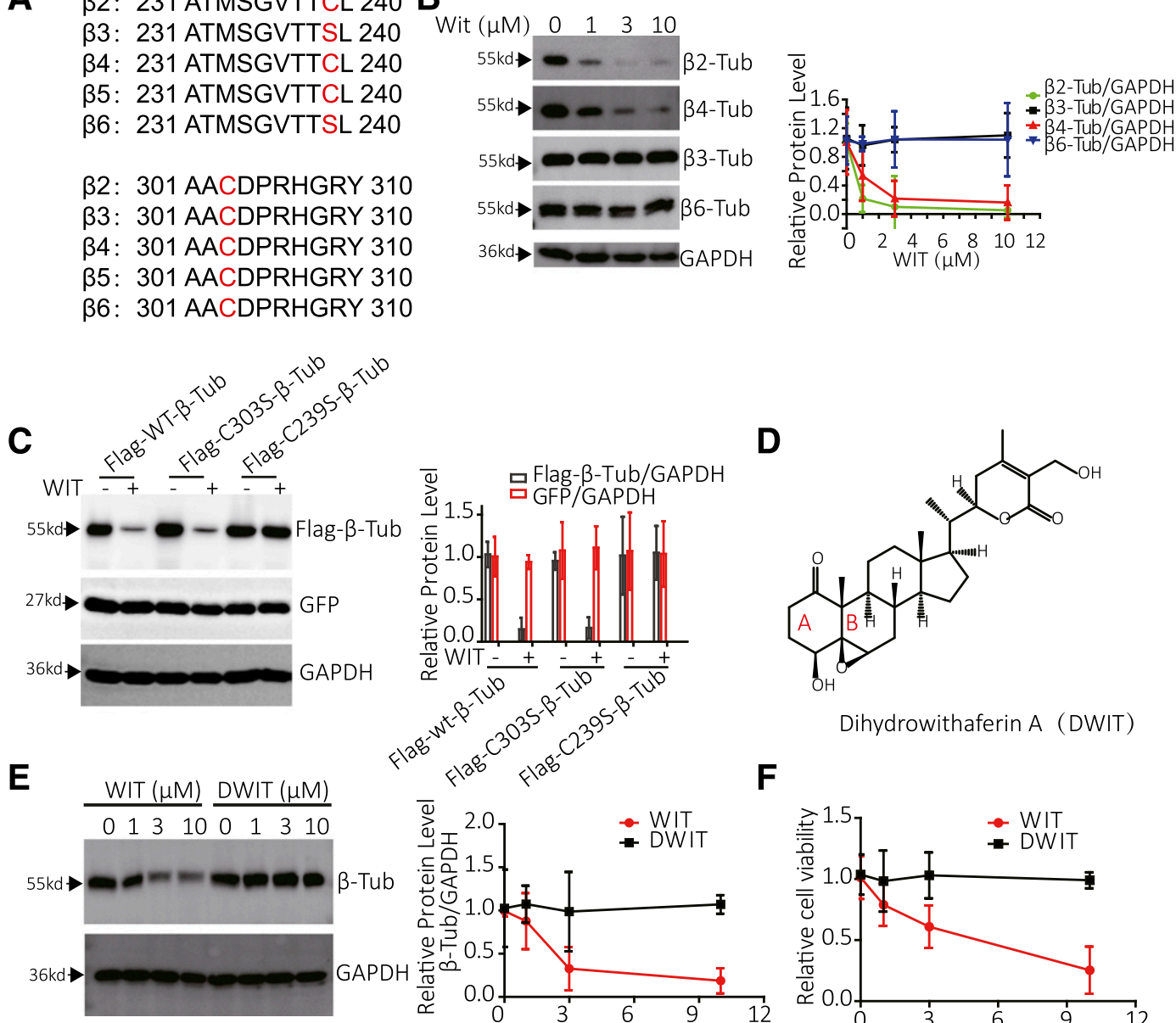

D

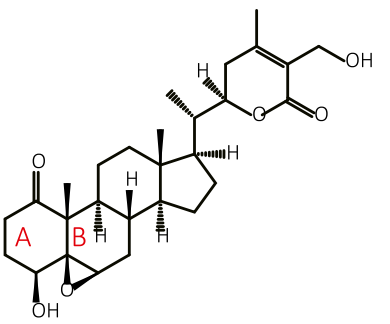

Dihydrowithaferin A (DWIT)

F
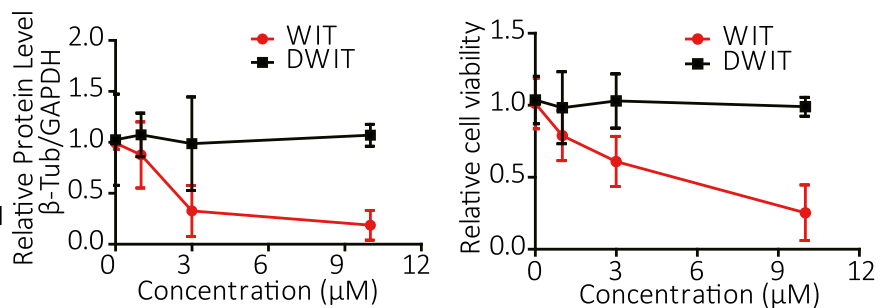

Fig. 5. Covalent binding between WIT and Cys239 accounts for WIT-induced tubulin degradation. (A) Selected amino acid sequences of $\beta 2-, \beta 3-, \beta 4-, \beta 5$ $(\beta)$-, and $\beta 6$-tubulin isoforms. (B) HeLa cells were treated with increasing concentrations of WIT for 16 hours, and $\beta 2-, \beta 3-, \beta 4-$ and $\beta 6$-tubulin protein levels were detected via Western blot. The right image depicts quantitative data on $\beta 2-, \beta 3-, \beta 4-$, and $\beta 6$-tubulin protein expression. Values are presented as mean $\pm 95 \%$ CI of three independent experiments. (C) HeLa cells were transiently transfected with MSCV-IRES-GFP vectors coexpressing GFP and WT-FLAG- $\beta$-Tubulin, C239S-FLAG- $\beta$-Tubulin, or C303S-FLAG- $\beta$-Tubulin for 24 hours. Cells were treated with or without $3 \mu$ M WIT for 16 hours, and GFP and FLAG-tubulin detected via Western blot. The right image depicts quantitative data on GFP and FLAG-tubulin. Values are presented as mean \pm 95\% CI of three independent experiments. (D) Chemical structure of dihydrowithaferin A (DWIT). (E) HeLa cells were treated with increasing concentrations of WIT or DWIT for 16 hours, and $\beta$-tubulin protein was detected via Western blot. The right image depicts quantitative data on $\beta$-tubulin protein. Values are presented as mean $\pm 95 \% \mathrm{CI}$ of three independent experiments. (F) HeLa cells were treated with increasing concentrations of WIT or DWIT for 48 hours, and cell viability was assessed with the trypan blue exclusion assay.Values are presented as mean $\pm 95 \%$ CI of three independent experiments. $\beta$-Tub, $\beta$-tubulin; $\beta 2$-Tub, $\beta 2$-tubulin; $\beta 3$-Tub, $\beta$-3tubulin; $\beta 4$-Tub, $\beta 4$-tubulin; $\beta 6$-Tub, $\beta 6$-tubulin.

Tubulin remains an important target for the development of stable and efficient antitumor drugs, investigation of mechanisms underlying tumor progression, and clinical research (Dumontet and Jordan, 2010).

The development of microtubule-targeted drugs has attracted significant commercial investment. Functionally, tubulin binders can be divided into two categories: polymerization agents (such as paclitaxel) and depolymerization agents (such as vinblastine) (Ravelli et al., 2004; Gigant et al., 2005; Ojeda-Lopez et al., 2014). However, the tubulin inhibitors identified to date have no effects on tubulin protein levels. Previous experiments by our group showed that small molecules covalently bound to the Cys239 site, including T0070906, T007-1, and T138067, promoting tubulin degradation (Yang et al., 2019). These small molecules were collectively classified as the third type of tubulin inhibitor-tubulin degradation agent.

In this study, we identified WIT as another naturally derived tubulin-degrading agent that also forms a covalent bond with cysteine at position 239 of $\beta$-tubulin. Tubulin degradation agents with distinct mechanisms of action should provide more options for the design of effective tubulin-based drugs and facilitate in-depth analysis of mechanisms underlying tubulin degradation.

Target protein degradation technology is a clinically effective treatment strategy-for instance, the application of fulvestrant-induced estrogen receptor degradation for breast cancer (Wu et al., 2005) and all-trans retinoic acid-induced retinoic acid receptor degradation for promyelocytic leukemia therapy (Zhu et al., 1999). The newly developed proteolysis targeting chimera (PROTAC) protein degradation technology plays a significant part in modern anticancer drug design (Lai and Crews, 2017). However, PROTACs are made up of two small molecules, which is an inherent disadvantage because the molecular weight is usually large and pharmacokinetic data as well as drug-forming properties are poor (Lai and Crews, 2017). So far, no small molecule-based PROTAC has 
been developed for degradation of tubulin. Experiments from our current study support covalent combination with Cys239 as an effective strategy to promote tubulin degradation with good development prospects; because the molecular weight is small, drugs with high-quality pharmacokinetic data could be designed.

The unsaturated double bond of the A ring of WIT is important for covalent bond formation. Our studies clearly indicate that the unsaturated double bond is essential for WIT binding to tubulin because DWIT devoid of the unsaturated double bond in the A ring showed total loss of tubulin degradation and antiproliferation activity. Based on these findings, we conclude that the covalent bond is necessary for both binding and degradation activities.

Previous studies have additionally reported covalent binding of WIT to Cys303 located on the surface of $\beta$-tubulin (Antony et al., 2014). In this case, the unsaturated double bond of the A ring of WIT that can easily form a covalent bond is almost completely exposed, and steric hindrance is small, allowing reaction with the cysteine residue on the protein surface, which leads to the possibility that the covalent bond between WIT and Cys303 is due to a nonspecific reaction. Substitution of cysteine with serine at position 303 had no effect on the degradation activity of WIT, indicative of no pharmacological effect of the WIT-Cys303 bond. However, further studies are required to ascertain the potential biologic effects of the WITCys303 bond.

Moreover, WIT covalently interacted with Cys239 of $\beta$-tubulin located near the colchicine site. A competition assay using colchicine site inhibitors, such as colchicine, nocodazole, and plinabulin, blocked WIT-induced tubulin degradation, clearly indicating that WIT binding to the colchicine site accounts for its tubulin degradation activity. Most importantly, substitution of cysteine with serine at position 239 abolished the degradation activity of WIT, further supporting the theory that formation of the WIT-Cys239 bond is a prerequisite for WIT-mediated degradation.

Of note, WIT also promotes the degradation of $\alpha$-tubulin. The underlying mechanism is proposed as both $\alpha$ - and $\beta$-tubulin are only stable in dimers in the cell and clearance of $\beta$-tubulin by WIT will cause lots of unstable monomeric $\alpha$-tubulin, which could be easily degraded by proteasome. However, this is only a theoretical assumption and requires further investigation.

Our collective findings demonstrate that the natural compound WIT forms a covalent bond with Cys239 of $\beta$-tubulin to promote tubulin degradation, providing another example of a covalent modifier of Cys at position 239 of $\beta$-tubulin as an effective tubulin degradation agent with therapeutic potential.

\section{Authorship Contributions}

Participated in research design: Yang, Chen.

Conducted experiments: Yang, Yan, Li, Niu

Contributed new reagents or analytic tools: Yang, Yan, Li.

Performed data analysis: Yang.

Wrote or contributed to the writing of the manuscript: Yang, Ye, Chen.

Note Added in Proof-Research supported by China Postdoctoral Science Foundation 2019T120855 was accidentally not included in the Fast Forward version published October 4, 2019. The funding footnote has now been corrected.

\section{References}

Antony ML, Lee J, Hahm ER, Kim SH, Marcus AI, Kumari V, Ji X, Yang Z, Vowell CL, Wipf P, et al. (2014) Growth arrest by the antitumor steroidal lactone Withaferin A in human breast cancer cells is associated with down-regulation and covalent binding at cysteine 303 of $\beta$-tubulin. $J$ Biol Chem 289:1852-1865.

Balaguer FA, Mühlethaler T, Estévez-Gallego J, Calvo E, Giménez-Abián JF, Risinger AL, Sorensen EJ, Vanderwal CD, Altmann KH, Mooberry SL, et al. (2019) Crystal structure of the cyclostreptin-tubulin adduct: implications for tubulin activation by taxane-site ligands. Int J Mol Sci 20:1392.

Bargagna-Mohan P, Hamza A, Kim YE, Khuan Abby Ho Y, Mor-Vaknin N, Wendschlag N, Liu J, Evans RM, Markovitz DM, Zhan C-G, et al. (2007) The tumor inhibitor and antiangiogenic agent Withaferin A targets the intermediate filament protein vimentin. Chem Biol 14:623-634.

Buey RM, Calvo E, Barasoain I, Pineda O, Edler MC, Matesanz R, Cerezo G, Vanderwal CD, Day BW, Sorensen EJ, et al. (2007) Cyclostreptin binds covalently to microtubule pores and lumenal taxoid binding sites. Nat Chem Biol 3:117-125.

Drahl C, Cravatt BF, and Sorensen EJ (2005) Protein-reactive natural products. Angew Chem Int Ed Engl 44:5788-5809.

Dumontet C and Jordan MA (2010) Microtubule-binding agents: a dynamic field of cancer therapeutics. Nat Rev Drug Discov 9:790-803.

Ernest H (2008) An overview of compounds that interact with tubulin and their effects on microtubule assembly, in The Role of Microtubules in Cell Biology, Neurobiology, and Oncology (Fojo T ed) pp 1-20, Humana Press, Totowa, NJ.

Falsey RR, Marron MT, Gunaherath GM, Shirahatti N, Mahadevan D, Gunatilaka $\mathrm{AA}$, and Whitesell L (2006) Actin microfilament aggregation induced by Withaferin A is mediated by annexin II. Nat Chem Biol 2:33-38.

Fortin S, Lacroix J, Côté MF, Moreau E, Petitclerc E, and C-Gaudreault R (2010) Quick and simple detection technique to assess the binding of antimicrotubule agents to the colchicine-binding site. Biol Proced Online 12:113-117.

Gambhir L, Checker R, Sharma D, Thoh M, Patil A, Degani M, Gota V, and Sandur SK (2015) Thiol dependent NF- $\kappa$ B suppression and inhibition of T-cell mediated adaptive immune responses by a naturally occurring steroidal lactone Withaferin A. Toxicol Appl Pharmacol 289:297-312.

Gigant B, Wang C, Ravelli RB, Roussi F, Steinmetz MO, Curmi PA, Sobel A, and Knossow M (2005) Structural basis for the regulation of tubulin by vinblastine. Nature 435:519-522.

Gu M, Yu Y, Gunaherath GM, Gunatilaka AA, Li D, and Sun D (2014) Structureactivity relationship (SAR) of withanolides to inhibit Hsp90 for its activity in pancreatic cancer cells. Invest New Drugs 32:68-74.

Heyninck K, Lahtela-Kakkonen M, Van der Veken P, Haegeman G, and Vanden Berghe W (2014) Withaferin A inhibits NF- $\kappa$ B activation by targeting cysteine 179 in IKK $\beta$. Biochem Pharmacol 91:501-509.

Khan SA, Khan SB, Shah Z, and Asiri AM (2016) Withanolides: biologically active constituents in the treatment of Alzheimer's disease. Med Chem 12:238-256.

Kupchan SM, Anderson WK, Bollinger P, Doskotch RW, Smith RM, Renauld JA, Schnoes HK, Burlingame AL, and Smith DH (1969) Tumor inhibitors. XXXIX. Active principles of Acnistus arborescens. Isolation and structural and spectral studies of Withaferin A and withacnistin. J Org Chem 34:3858-3866.

Lai AC and Crews CM (2017) Induced protein degradation: an emerging drug discovery paradigm. Nat Rev Drug Discov 16:101-114.

Lee J, Liu J, Feng X, Salazar Hernández MA, Mucka P, Ibi D, Choi JW, and Ozcan U (2016) Withaferin A is a leptin sensitizer with strong antidiabetic properties in mice. Nat Med 22:1023-1032.

Maitra R, Porter MA, Huang S, and Gilmour BP (2009) Inhibition of $\mathrm{NF}_{\kappa} \mathrm{B}$ by the natural product Withaferin A in cellular models of cystic fibrosis inflammation. J Inflamm (Lond) 6:15.

Mohan R, Hammers HJ, Bargagna-Mohan P, Zhan XH, Herbstritt CJ, Ruiz A, Zhang L, Hanson AD, Conner BP, Rougas J, et al. (2004) Withaferin A is a potent inhibitor of angiogenesis. Angiogenesis 7:115-122.

Munagala R, Kausar H, Munjal C, and Gupta RC (2011) Withaferin A induces p53-dependent apoptosis by repression of HPV oncogenes and upregulation of tumor suppressor proteins in human cervical cancer cells. Carcinogenesis 32 : 1697-1705.

Na GC and Timasheff SN (1982) In vitro vinblastine-induced tubulin paracrystals. J Biol Chem 257:10387-10391.

Ojeda-Lopez MA, Needleman DJ, Song C, Ginsburg A, Kohl PA, Li Y, Miller HP, Wilson L, Raviv U, Choi MC, et al. (2014) Transformation of taxol-stabilized microtubules into inverted tubulin tubules triggered by a tubulin conformation switch. Nat Mater 13:195-203.

Prota AE, Bargsten K, Diaz JF, Marsh M, Cuevas C, Liniger M, Neuhaus C, Andreu JM, Altmann KH, and Steinmetz MO (2014a) A new tubulin-binding site and pharmacophore for microtubule-destabilizing anticancer drugs. Proc Natl Acad Sci USA 111:13817-13821.

Prota AE, Bargsten K, Northcote PT, Marsh M, Altmann K-H, Miller JH, Díaz JF, and Steinmetz MO (2014b) Structural basis of microtubule stabilization by laulimalide and peloruside A. Angew Chem Int Ed Engl 53:1621-1625.

Ravelli RB, Gigant B, Curmi PA, Jourdain I, Lachkar S, Sobel A, and Knossow M (2004) Insight into tubulin regulation from a complex with colchicine and a stathmin-like domain. Nature 428:198-202.

Ravindran R, Sharma N, Roy S, Thakur AR, Ganesh S, Kumar S, Devi J, and Rajkumar J (2015) Interaction studies of Withania somnifera's key metabolite Withaferin A with different receptors associated with cardiovascular disease. Curr Comput Aided Drug Des 11:212-221.

Ren J, Tang CZ, Li XD, Niu ZB, Zhang BY, Zhang T, Gao MJ, Ran XZ, Su YP, and Wang FC (2018) Identification of G2/M phase transition by sequential nuclear and cytoplasmic changes and molecular markers in mice intestinal epithelial cells. Cell Cycle 17:780-791.

Siddique AA, Joshi P, Misra L, Sangwan NS, and Darokar MP (2014) 5,6-De-epoxy-5en-7-one-17-hydroxy withaferin A, a new cytotoxic steroid from Withania somnifera L. Dunal leaves. Nat Prod Res 28:392-398. 
Stanton RA, Gernert KM, Nettles JH, and Aneja R (2011) Drugs that target dynamic microtubules: a new molecular perspective. Med Res Rev 31:443-481.

Tekula S, Khurana A, Anchi P, and Godugu C (2018) Withaferin-A attenuates multiple low doses of streptozotocin (MLD-STZ) induced type 1 diabetes. Biomed Pharmacother 106:1428-1440.

Thomas NE, Thamkachy R, Sivakumar KC, Sreedevi KJ, Louis XL, Thomas SA, Kumar R, Rajasekharan KN, Cassimeris L, and Sengupta S (2014) Reversible action of diaminothiazoles in cancer cells is implicated by the induction of a fast conformational change of tubulin and suppression of microtubule dynamics. Mol Cancer Ther 13:179-189.

Vanden Berghe W, Sabbe L, Kaileh M, Haegeman G, and Heyninck K (2012) Molecular insight in the multifunctional activities of Withaferin A. Biochem Pharmacol 84:1282-1291.

Wu YL, Yang X, Ren Z, McDonnell DP, Norris JD, Willson TM, and Greene GL (2005) Structural basis for an unexpected mode of SERM-mediated ER antagonism. Mol Cell 18:413-424

Yan W, Yang T, Yang J, Wang T, Yu Y, Wang Y, Chen Q, Bai P, Li D, Ye H, et al (2018) SKLB060 reversibly binds to colchicine site of tubulin and possesses efficacy in multidrug-resistant cell lines. Cell Physiol Biochem 47:489-504.

Yang J, Li Y, Yan W, Li W, Qiu Q, Ye H, and Chen L (2019) Covalent modification of Cys-239 in $\beta$-tubulin by small molecules as a strategy to promote tubulin heterodimer degradation. J Biol Chem 294:8161-8170.
Yang J, Wang Y, Wang T, Jiang J, Botting CH, Liu H, Chen Q, Yang J, Naismith JH, Zhu X, et al. (2016) Pironetin reacts covalently with cysteine-316 of $\alpha$-tubulin to destabilize microtubule. Nat Commun 7:12103.

Yang J, Yan W, Yu Y, Wang Y, Yang T, Xue L, Yuan X, Long C, Liu Z, Chen X, et al. (2018) The compound millepachine and its derivatives inhibit tubulin polymerization by irreversibly binding to the colchicine-binding site in $\beta$-tubulin. $J$ Biol Chem 293:9461-9472.

Yu Y, Hamza A, Zhang T, Gu M, Zou P, Newman B, Li Y, Gunatilaka AA, Zhan C-G, and Sun D (2010) Withaferin A targets heat shock protein 90 in pancreatic cancer cells. Biochem Pharmacol 79:542-551.

Zhu J, Gianni M, Kopf E, Honoré N, Chelbi-Alix M, Koken M, Quignon F, RochetteEgly C, and de Thé H (1999) Retinoic acid induces proteasome-dependent degradation of retinoic acid receptor alpha $(\operatorname{RAR} \alpha)$ and oncogenic $\operatorname{RAR} \alpha$ fusion proteins. Proc Natl Acad Sci USA 96:14807-14812.

Address correspondence to: Lijuan Chen, State Key Laboratory of Biotherapy and Cancer Center, West China Hospital of Sichuan University and Collaborative Innovation Center of Biotherapy and Cancer, Chengdu 610041, People's Republic of China. E-mail:chenlijuan125@163.com 